\title{
Importancia de las lagunas temporales para la conservación de la biodiversidad de artrópodos edáficos en zonas agrícolas de Castilla-la Mancha
}

\author{
García Ruiz, Andrés \\ Departamento de Didácticas Específicas. Facultad de Formación de Profesorado y de educación. \\ Universidad Autónoma de Madrid.28049 Madrid. E-mail: andres.garcia.ruiz@uam.es
}

\section{RESUMEN}

El presente trabajo tiene como objeto el estudio comparativo de las comunidades de artrópodos edáficos en dos lagunas temporales de Castilla-La Mancha, empleándose trampas de caída de acción continua. En las zonas de estudio se distinguieron áreas con diferentes características ambientales.

Se realizaron estudios de frecuencia, abundancia, riqueza y análisis multivariante para establecer las preferencias de los grupos encontrados por determinados hábitats.

Con los resultados obtenidos podemos señalar que las lagunas temporales favorecen la conservación y biodiversidad de determinados grupos de artrópodos edáficos.

Palabras clave: artrópodos edáficos, lagunas temporales, Castilla-La Mancha.

\begin{abstract}
The aim of this work is the comparative study of the soil arthropod communities in two transient lagoons from Castilla-La Mancha, by the use of pit-fall type traps. In the study areas sites with different environmental characteristics were identified. Analysis of frequency, abundance and richness and multivariate analyses were performed to detect preferences for particular habitats among the groups found.

The results show that the transient lagoons favour the conservation and biodiversity of certain groups of edaphic arthropods.
\end{abstract}

Keywords: edaphic arthropods, shallow lakes, Castilla-La Mancha.

\section{INTRODUCCIÓN}

La biodiversidad de los humedales se sigue considerando un atributo al que contribuyen mucho la avifauna, la vegetación y biocenosis macroscópicas más o menos vistosas, mientras que la mayor fuente de biodiversidad son seguramente los artrópodos, que siempre están presentes en los humedales, inundados o secos, alterados o conservados, en periodos de inundación efímera de los de uno cada diez años igual que en los periodos secos intermedios, sin olvidar su función en los ciclos biogeoquímicos de los humedales, pues constituyen eslabones tróficos fundamentales para las vías de reciclado de la producción primaria tan alta que se atribuye a estos sistemas.

La entomofauna palustre está constituida por comunidades que podrían considerarse actualmente reliquias de la fauna colonizadora de las grandes cuencas palustres y lacustres de finales del Neógeno y debido al papel que tienen los artrópodos edáficos en el mantenimiento de la biodiversidad global de los humedales, esto le concede especial interés para el 
estudio y conocimiento de la biodiversidad y ecología de los escasos humedales naturales que subsisten en la Península Ibérica.

Cabe destacar la influencia que diferentes factores externos ejercen sobre el sistema edáfico, considerándose algunos grupos de artrópodos como indicadores de la posible influencia externa, principalmente las deforestaciones, zonas alternativas de pastoreo y manejo agrícola (Hagvar, 1982; Poursin \& Ponge, 1984).

\section{MATERIAL Y MÉTODOS}

\section{Zona de estudio}

La zona de estudio se encuentra ubicada en el término municipal de Moral de Calatrava, situado en la parte central de la provincia de Ciudad Real e integrado en la comarca del Campo de Calatrava. En él existe un complejo lagunar constituido por El Calderón, La Laguna y el Salobral. Esta última no se ha estudiado por no tener un carácter natural, debido a que se nutre de aguas procedentes de la depuradora del municipio. Todas ellas son de origen volcánico, es decir, restos de antiguos cráteres explosivos y sufren, como todas las zonas húmedas de la meseta, una marcada estacionalidad. Estas lagunas fueron desecadas en su día para implantar en ellas cultivos agrícolas.

Debido a las abundantes lluvias caídas durante 1995 y 1996, se inundaron las dos lagunas objeto de estudio, resurgiendo la orla de vegetación lacustre en su perímetro.

\section{Toma de muestras}

Para la captura del material se usaron trampas de caída de acción continua, también conocidas como trampas de paso o pit-fall, sistema apto para la captura de fauna epigea. Cada trampa consiste en un recipiente troncocónico de $13 \mathrm{~cm}$. de altura y 12 y $9 \mathrm{~cm}$. de diámetro superior e inferior respectivamente, conteniendo agua y etilenglicol en proporción 10:1, para evitar la evaporación del agua y facilitar la conservación del material capturado (Ascaso, 1984).

En cada una de las lagunas de estudio se distinguieron tres áreas: dos zonas dedicadas al cultivo: viña y olivar y una tercera liega, es decir sin cultivar. En cada área se instalaron cinco trampas, separadas cinco metros una de otra y colocadas en línea recta, paralela a la linea de agua, recogiendo el material a los tres días. Los muestreos sucesivos se realizaron manteniendo las trampas en los mismos puntos que el primero.

La recolección del material faunístico se realizó con periodicidad trimestral y las muestras fueron tomadas estacionalmente en enero, abril, agosto y noviembre de 1995, 1996, 1998 y 1999, correspondiendo los dos primeros años a épocas de elevada pluviosidad y los dos segundos a periodos de escasas precipitaciones.

\section{Métodos cuantitativos}

\section{Frecuencia y abundancia relativa y riqueza especifica}

Después de haber realizado el estudio faunístico de los individuos capturados, se hizo un análisis de abundancia relativa (porcentaje del número de individuos de cada taxón con respecto del total capturado en cada hábitat) y frecuencia relativa (porcentaje del número de muestreos en que se han encontrado ejemplares del grupo con respecto del total de muestreos realizados) de los diferentes grupos de artrópodos edáficos encontrados en cada una de las zonas de estudio de las dos lagunas estudiadas. Según el valor de la abundancia relativa y siguiendo la delimitación de Krogerus (Ascaso, 1986), determinaremos la dominancia de los diferentes grupos de artrópodos, considerandose como grupos dominantes todos los que presenten una abundancia relativa superior al $5 \%$.

Para caracterizar a los diferentes grupos hemos utilizado el coeficiente de Tischler (Ascaso, 1986), que los clasifica dependiendo de su frecuencia.

Para estudiar la distribución horizontal de las comunidades de artrópodos edáficos en las 
zonas de estudio, hemos aplicado el índice de Morosita (Cancela da Fonseca, 1966), el cual dependiendo de la relación entre la varianza y la media aritmética de la densidad de población en las diferentes muestras, nos da una idea de los tipos de distribución horizontal de los organismos, obteniendo con las densidades calculadas en las áreas de estudio que la distribución que siguen todos los grupos de artrópodos es en agregados, es decir la varianza es mayor que la media aritmética.

Con el objetivo de poder caracterizar la biodiversidad de artrópodos de cada una de las zonas estudiadas, hemos calculado la riqueza específica.

\section{Análisis multivariante}

Con los datos obtenidos, utilizando el programa informático SPSS, realizamos análisis de cluster para determinar las afinidades entre las distintas muestras con el fin de identificar diferencias entre muestreos y entre biótopos estudiados. Hemos realizado los dendrogramas correspondientes a las clasificaciones jerárquicas de los taxones encontrados en los muestreos "Biótopos-Año", construidos en base a los valo-

\section{$\mathrm{N}^{\circ}$ de artrópodos}
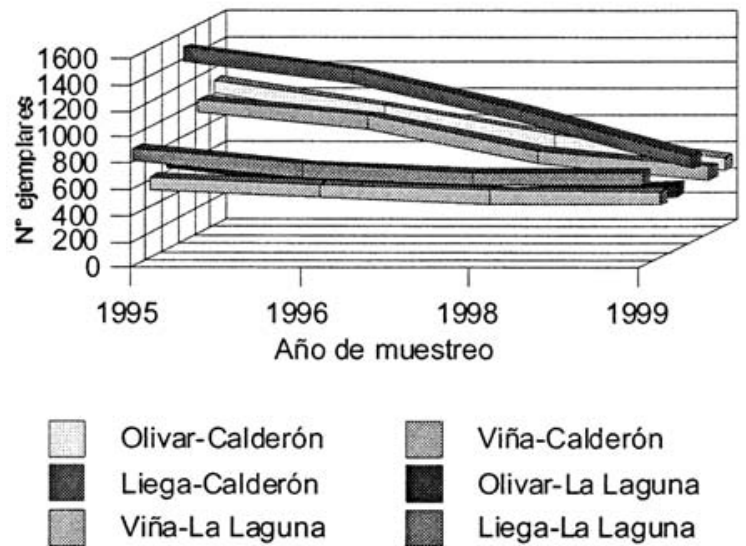

Figura 1. Número de artrópodos capturados por hábitat y año en las dos lagunas estudiadas. Number of arthropods captured by habitat and year in the two studied lagoons.

res ínter-grupo, aplicando el índice de correlación de Pearson y finalmente también hemos realizado un análisis de cluster, aplicando el índice de correlación de Pearson a la matriz de grupos obtenidos en las dos lagunas, durante los cuatro años de muestreo.

Tabla 1. Abundancia relativa de artrópodos en Calderón. Relative abundance of arthropods in Calderón.

\begin{tabular}{|c|c|c|c|c|c|c|c|c|c|c|c|c|}
\hline \multicolumn{13}{|c|}{ Calderón } \\
\hline Año & & 1995 & & & 1996 & & & 1998 & & & 1999 & \\
\hline Grupo/hábitat & Olivar & Viña & Liega & Olivar & Viña & Liega & Olivar & Viña & Liega & Olivar & Viña & Liega \\
\hline Colémbolos & 0.39 & 0.24 & 0.58 & 0.44 & 0.27 & 0.57 & 0.48 & 0.23 & 0.59 & 0.43 & 0.18 & 0.43 \\
\hline Coleópteros & 0.02 & 0.01 & 0.02 & 0.02 & 0.01 & 0.01 & 0.02 & 0.00 & 0.02 & 0.03 & 0.01 & 0.02 \\
\hline Dípteros & 0.06 & 0.00 & 0.02 & 0.05 & 0.00 & 0.02 & 0.06 & 0.00 & 0.02 & 0.02 & 0.00 & 0.03 \\
\hline Dictiópteros & 0.00 & 0.00 & 0.00 & 0.00 & 0.00 & 0.00 & 0.00 & 0.00 & 0.00 & 0.00 & 0.00 & 0.00 \\
\hline Efemerópteros & 0.00 & 0.00 & 0.00 & 0.00 & 0.00 & 0.00 & 0.00 & 0.00 & 0.00 & 0.00 & 0.00 & 0.00 \\
\hline Hemípteros & 0.00 & 0.00 & 0.00 & 0.01 & 0.00 & 0.00 & 0.00 & 0.00 & 0.00 & 0.00 & 0.00 & 0.00 \\
\hline Himenópteros & 0.20 & 0.02 & 0.02 & 0.13 & 0.03 & 0.02 & 0.15 & 0.02 & 0.04 & 0.15 & 0.04 & 0.07 \\
\hline Tisanópteros & 0.00 & 0.00 & 0.03 & 0.00 & 0.00 & 0.03 & 0.00 & 0.00 & 0.03 & 0.00 & 0.00 & 0.00 \\
\hline Ortópteros & 0.00 & 0.00 & 0.00 & 0.00 & 0.00 & 0.00 & 0.00 & 0.00 & 0.00 & 0.00 & 0.00 & 0.00 \\
\hline Odonatos & 0.03 & 0.02 & 0.01 & 0.02 & 0.01 & 0.00 & 0.00 & 0.00 & 0.00 & 0.00 & 0.00 & 0.00 \\
\hline Ácaros & 0.23 & 0.65 & 0.24 & 0.26 & 0.63 & 0.25 & 0.22 & 0.64 & 0.21 & 0.27 & 0.59 & 0.33 \\
\hline Arañas & 0.19 & 0.01 & 0.02 & 0.01 & 0.01 & 0.03 & 0.03 & 0.03 & 0.03 & 0.04 & 0.02 & 0.05 \\
\hline Pseudoescorpiones & 0.00 & 0.00 & 0.00 & 0.00 & 0.00 & 0.00 & 0.00 & 0.00 & 0.00 & 0.00 & 0.00 & 0.00 \\
\hline Crustáceos & 0.00 & 0.00 & 0.00 & 0.00 & 0.00 & 0.00 & 0.00 & 0.00 & 0.00 & 0.01 & 0.01 & 0.00 \\
\hline Miriápodos & 0.00 & 0.00 & 0.00 & 0.00 & 0.00 & 0.00 & 0.00 & 0.00 & 0.00 & 0.01 & 0.01 & 0.01 \\
\hline
\end{tabular}




\section{RESULTADOS}

Se capturaron 17136 animales correspondientes a 15 grupos de artrópodos edáficos, de los cuales 10178 corresponden a la laguna de Calderón y 6958 a La Laguna.

El número de individuos más elevado recogido en Calderón (6543) corresponde a los años de mayor pluviosidad, frente a los 3725 capturados durante 1998 y 1999, al igual que ocurre en La Laguna (3865 y 3093, respectivamente) (Fig. 1).

Por hábitats, la zona liega es la que presenta mayor número de ejemplares en las dos zonas estudiadas, presentando por ello las zonas naturales mayor número de individuos que las modificadas para uso agrícola.

Respecto a los diferentes taxones, los colémbolos son los más abundantes en Calderón, presentando los valores más elevados la zona liega, seguido de los ácaros, que muestran una clara preferencia por la zona dedicada al cultivo de la vid, y de los himenópteros, que dominan en el olivar. En La Laguna ocurre igual que en el caso anterior, los colémbolos son los más numerosos en la zona liega, seguido de los ácaros en la viña y los himenópteros en el olivar.
Ácaros y colémbolos son los artrópodos edáficos más abundantes en ambas lagunas (Tablas 1 y 2). En Calderón presentan porcentajes de abundancia muy similares en las diferentes zonas de estudio, durante los cuatro años de muestreo, al igual que ocurre en La Laguna.

Con los valores de abundancia relativa obtenidos, podemos señalar que en la laguna de Calderón y concretamente en la parcela del olivar no encontramos ningún grupo dominante; en la viña los ácaros son los dominantes y en la zona natural los colémbolos.

Para La Laguna, al igual que en el caso anterior en el olivar no existe ningún grupo con categoría dominante; en la viña dominan los ácaros excepto en 1996 y en la zona liega los colémbolos en los dos últimos años.

Analizando las poblaciones de los grupos más importantes y su variación a lo largo del año, observamos como ácaros y colémbolos son los únicos grupos que hemos encontrado durante todo el año (Tablas 3 y 4). En el resto de los grupos podemos observar en la mayoría de ellos una marcada estacionalidad, ya que muchos de ellos han sido capturados sólo en dos estaciones del año.

Tabla 2. Abundancia relativa de artrópodos en La Laguna. Relative abundance of arthropods in La Laguna.

\begin{tabular}{|c|c|c|c|c|c|c|c|c|c|c|c|c|}
\hline \multicolumn{13}{|c|}{ La Laguna } \\
\hline Año & & 1995 & & & 1996 & & & 1998 & & & 1999 & \\
\hline Grupo/hábitat & Olivar & Viña & Liega & Olivar & Viña & Liega & Olivar & Viña & Liega & Olivar & Viña & Liega \\
\hline Colémbolos & 0.32 & 0.25 & 0.49 & 0.36 & 0.34 & 0.47 & 0.30 & 0.29 & 0.55 & 0.26 & 0.27 & 0.55 \\
\hline Coleópteros & 0.02 & 0.02 & 0.02 & 0.07 & 0.00 & 0.02 & 0.03 & 0.04 & 0.03 & 0.03 & 0.03 & 0.03 \\
\hline Dípteros & 0.07 & 0.00 & 0.03 & 0.23 & 0.00 & 0.03 & 0.02 & 0.00 & 0.01 & 0.01 & 0.00 & 0.00 \\
\hline Dictiópteros & 0.00 & 0.00 & 0.00 & 0.00 & 0.00 & 0.00 & 0.00 & 0.00 & 0.00 & 0.00 & 0.00 & 0.00 \\
\hline Efemerópteros & 0.00 & 0.00 & 0.00 & 0.00 & 0.00 & 0.00 & 0.00 & 0.00 & 0.00 & 0.00 & 0.00 & 0.00 \\
\hline Hemípteros & 0.01 & 0.00 & 0.01 & 0.04 & 0.00 & 0.02 & 0.00 & 0.00 & 0.00 & 0.00 & 0.00 & 0.00 \\
\hline Himenópteros & 0.15 & 0.19 & 0.01 & 0.26 & 0.04 & 0.01 & 0.11 & 0.03 & 0.02 & 0.15 & 0.02 & 0.02 \\
\hline Tisanópteros & 0.00 & 0.00 & 0.03 & 0.00 & 0.00 & 0.03 & 0.00 & 0.00 & 0.03 & 0.00 & 0.00 & 0.03 \\
\hline Ortópteros & 0.00 & 0.00 & 0.00 & 0.00 & 0.00 & 0.00 & 0.00 & 0.00 & 0.00 & 0.00 & 0.00 & 0.00 \\
\hline Odonatos & 0.04 & 0.03 & 0.02 & 0.16 & 0.00 & 0.02 & 0.00 & 0.00 & 0.00 & 0.00 & 0.00 & 0.00 \\
\hline Ácaros & 0.31 & 0.60 & 0.28 & 0.35 & 0.29 & 0.32 & 0.43 & 0.54 & 0.26 & 0.44 & 0.55 & 0.26 \\
\hline Arañas & 0.02 & 0.02 & 0.03 & 0.00 & 0.07 & 0.02 & 0.04 & 0.03 & 0.04 & 0.03 & 0.03 & 0.04 \\
\hline Pseudoescorpiones & 0.00 & 0.00 & 0.00 & 0.00 & 0.00 & 0.00 & 0.00 & 0.00 & 0.00 & 0.00 & 0.00 & 0.00 \\
\hline Crustáceos & 0.00 & 0.00 & 0.00 & 0.00 & 0.00 & 0.00 & 0.00 & 0.01 & 0.00 & 0.00 & 0.01 & 0.00 \\
\hline Miriápodos & 0.01 & 0.00 & 0.00 & 0.00 & 0.04 & 0.01 & 0.02 & 0.01 & 0.01 & 0.02 & 0.01 & 0.01 \\
\hline
\end{tabular}


Tabla 3. Frecuencia relativa de artrópodos en Calderón. Relative frequency of arthropods in Calderón.

\begin{tabular}{|c|c|c|c|c|c|c|c|c|c|c|c|c|}
\hline \multicolumn{13}{|c|}{ Calderón } \\
\hline Año & & 1995 & & & 1996 & & & 1998 & & & 1999 & \\
\hline Grupo/hábitat & Olivar & Viña & Liega & Olivar & Viña & Liega & Olivar & Viña & Liega & Olivar & Viña & Liega \\
\hline Colémbolos & 1.00 & 1.00 & 1.00 & 1.00 & 1.00 & 1.00 & 1.00 & 1.00 & 1.00 & 1.00 & 1.00 & 1.00 \\
\hline Coleópteros & 0.50 & 0.50 & 0.50 & 0.50 & 0.50 & 0.50 & 0.50 & 0.25 & 0.50 & 0.50 & 0.25 & 0.50 \\
\hline Dípteros & 0.50 & 0.25 & 0.50 & 0.75 & 0.25 & 0.50 & 0.50 & 0.00 & 0.50 & 0.50 & 0.00 & 0.50 \\
\hline Dictiópteros & 0.00 & .000 & 0.25 & 0.00 & 0.00 & 0.00 & 0.00 & 0.00 & 0.00 & 0.00 & 0.00 & 0.00 \\
\hline Efemerópteros & 0.00 & 0.00 & 0.50 & 0.00 & 0.00 & 25 & 0.00 & 0.00 & 0.00 & 0.00 & 0.00 & 0.00 \\
\hline Hemípteros & 0.25 & 0.25 & 0.50 & 0.25 & 0.25 & 0.50 & 0.25 & 0.00 & 0.25 & 0.25 & 0.00 & 0.25 \\
\hline Himenópteros & 1.00 & 0.0 & 0.75 & 1.00 & 0.75 & 0.75 & 1.00 & 0.50 & 0.75 & 0.75 & 0.50 & 075 \\
\hline Tisanópteros & 0.25 & 0.25 & 0.50 & 0.25 & 0.25 & 0.75 & 0.00 & 0.25 & 0.50 & 0.00 & 0.25 & 0.25 \\
\hline Ortópteros & 0.25 & 0.00 & 0.00 & 0.25 & 0.25 & 0.25 & 0.00 & 0.00 & 0.00 & 0.00 & 0.00 & 0.00 \\
\hline Odonatos & 0.50 & 0.50 & 0.50 & 0.50 & 0.50 & 0.50 & 0.25 & 0.25 & 0.00 & 0.00 & 0.00 & 0.00 \\
\hline Ácaros & 1.00 & 1.00 & 1.00 & 1.00 & 1.00 & 1.00 & 1.00 & 1.00 & 1.00 & 1.00 & 1.00 & 1.00 \\
\hline Arañas & 0.50 & 0.50 & 0.75 & 0.50 & 0.50 & 0.75 & 0.50 & 0.50 & 0.50 & 0.75 & 0.75 & 0.75 \\
\hline Pseudoescorpiones & 0.00 & 0.00 & 0.00 & 0.25 & 0.00 & 0.00 & 0.00 & 0.25 & 0.00 & 0.00 & 0.00 & 0.00 \\
\hline Crustáceos & 0.25 & 0.00 & 0.50 & 0.50 & 0.00 & 0.25 & 0.25 & 0.25 & 0.50 & 0.25 & 0.25 & 0.25 \\
\hline Miriápodos & 0.25 & 0.25 & 0.50 & 0.25 & 0.00 & 0.50 & 0.50 & 0.50 & 0.25 & 0.50 & 0.50 & 0.50 \\
\hline
\end{tabular}

Respecto a la caracterización de los diferentes grupos, observamos que en Calderón los ácaros y colémbolos son euconstantes en las tres parcelas, es decir su frecuencia se encuentra entre
0.75 y 1 y en La Laguna estos grupos también presentan la misma categoría.

En el estudio de las densidades de los diferentes grupos en las dos lagunas, podemos

Tabla 4. Frecuencia relativa de artrópodos en La Laguna. Relative frequency of arthropods in La Laguna.

\begin{tabular}{|c|c|c|c|c|c|c|c|c|c|c|c|c|}
\hline \multicolumn{13}{|c|}{ La Laguna } \\
\hline Año & & 1995 & & & 1996 & & & 1998 & & & 1999 & \\
\hline Grupo/hábitat & Olivar & Viña & Liega & Olivar & Viña & Liega & Olivar & Viña & Liega & Olivar & Viña & Liega \\
\hline Colémbolos & 1.00 & 1.00 & 1.00 & 1.00 & 1.00 & 1.00 & 1.00 & 1.00 & 1.00 & 1.00 & 1.00 & 1.00 \\
\hline Coleópteros & 0.50 & 0.50 & 0.75 & 0.50 & 0.50 & 0.50 & 0.50 & 0.50 & 0.50 & 0.50 & 0.50 & 0.75 \\
\hline Dípteros & 0.50 & 0.25 & 0.50 & 0.50 & 0.25 & 0.50 & 0.50 & 0.25 & 0.25 & 0.25 & 0.25 & 0.25 \\
\hline Dictiópteros & 0.00 & 0.00 & 0.00 & 0.00 & 0.00 & 0.00 & 0.00 & 0.00 & 0.00 & 0.00 & 0.00 & 0.00 \\
\hline Efemerópteros & 0.00 & 0.00 & 0.50 & 0.00 & 0.00 & 0.25 & 0.00 & 0.00 & 0.00 & 0.00 & 0.00 & 0.00 \\
\hline Hemípteros & 0.25 & 0.25 & 0.50 & 0.25 & 0.25 & 0.50 & 0.25 & 0.00 & 0.25 & 0.25 & 0.00 & 0.25 \\
\hline Himenópteros & 0.75 & 0.50 & 0.50 & 0.75 & 0.25 & 0.50 & 0.50 & 0.50 & 0.50 & 0.75 & 0.50 & 0.50 \\
\hline Tisanópteros & 0.00 & 0.25 & 0.50 & 0.00 & 0.00 & 0.50 & 0.00 & 0.25 & 0.50 & 0.00 & 0.25 & 0.50 \\
\hline Ortópteros & 0.00 & 0.00 & 0.25 & 0.00 & 0.00 & 0.50 & 0.00 & 0.25 & 0.25 & 0.00 & 0.25 & 0.25 \\
\hline Odonatos & 0.50 & 0.50 & 0.50 & 0.50 & 0.50 & 0.25 & 0.00 & 0.00 & 0.00 & 0.25 & 0.25 & 0.00 \\
\hline Ácaros & 1.00 & 1.00 & 1.00 & 1.00 & 1.00 & 1.00 & 1.00 & 1.00 & 1.00 & 1.00 & 1.00 & 1.00 \\
\hline Arañas & 0.50 & 0.50 & 0.75 & 0.50 & 0.50 & 0.75 & 0.50 & 0.75 & 0.75 & 0.50 & 0.50 & 0.75 \\
\hline Pseudoescorpiones & 0.25 & 0.00 & 0.00 & 0.00 & 0.00 & 0.00 & 0.25 & 0.00 & 0.00 & 0.25 & 0.00 & 0.00 \\
\hline Crustáceos & 0.50 & 0.25 & 0.50 & 0.25 & 0.50 & 0.50 & 0.25 & 0.25 & 0.50 & 0.25 & 0.50 & 0.50 \\
\hline Miriápodos & 0.50 & 0.50 & 0.75 & 0.50 & 0.50 & 0.50 & 0.50 & 0.75 & 0.75 & 0.75 & 0.75 & 0.75 \\
\hline
\end{tabular}


Tabla 5. Densidades anuales de artrópodos en las dos lagunas de estudio. Annual densities of arthropods in the two studied lagoons.

\begin{tabular}{|c|c|c|c|c|c|c|c|c|}
\hline \multirow[b]{2}{*}{ Año/Grupo } & \multicolumn{4}{|c|}{ Calderón } & \multicolumn{4}{|c|}{ La Laguna } \\
\hline & 1995 & 1996 & 1998 & 1999 & 1995 & 1996 & 1998 & 1999 \\
\hline Colémbolos & 1179.5 & 1062.2 & 810.0 & 470.8 & 619.6 & 504.7 & 490.5 & 483.4 \\
\hline Coleópteros & 52.7 & 43.3 & 35.4 & 30.7 & 41.7 & 35.4 & 46.4 & 45.6 \\
\hline Dípteros & 85.0 & 69.2 & 51.9 & 26.7 & 66.1 & 63.7 & 16.5 & 14.1 \\
\hline Dictiópteros & 0.78 & 0.0 & 0.0 & 0.0 & 0.0 & 0.0 & 0.0 & 0.0 \\
\hline Efemerópteros & 10.2 & 7.08 & 0.0 & 0.0 & 5.51 & 3.14 & 0.0 & 0.0 \\
\hline Hemípteros & 17.3 & 20.47 & 7.08 & 4.72 & 15.7 & 22.0 & 4.72 & 4.72 \\
\hline Himenópteros & 224.4 & 140.9 & 122.0 & 109.4 & 105.5 & 70.0 & 63.7 & 79.5 \\
\hline Tisanópteros & 44.0 & 40.1 & 30.7 & 3.93 & 29.1 & 18.1 & 17.3 & 18.1 \\
\hline Ortópteros & 1.57 & 3.93 & 0.0 & 0.0 & 1.57 & 0.78 & 3.14 & 3.93 \\
\hline Odonatos & 58.2 & 35.4 & 3.93 & 0.0 & 55.1 & 51.1 & 0.0 & 4.72 \\
\hline Ácaros & 977.1 & 838.5 & 574.8 & 493.7 & 629.9 & 562.3 & 474.8 & 496.0 \\
\hline Arañas & 59.8 & 51.9 & 58.26 & 52.7 & 49.6 & 35.4 & 51.9 & 51.1 \\
\hline Pseudoescorpiones & 0.0 & 0.78 & 0.78 & 0.0 & 0.78 & 0.0 & 0.78 & 1.57 \\
\hline Crustáceos & 9.44 & 8.66 & 12.5 & 14.1 & 13.3 & 10.2 & 9.44 & 12.59 \\
\hline Miriápodos & 7.0 & 7.08 & 11.8 & 18.8 & 15.7 & 16.5 & 18.11 & 22.04 \\
\hline
\end{tabular}

observar como las densidades en ambas zonas de estudio presentan los valores más elevados de densidad durante los años de mayor grado de precipitaciones, y concretamente la presentan ácaros y colémbolos (Tabla 5).

En cuanto a la riqueza encontramos un comportamiento diferente entre las dos lagunas (Fig. 2); en Calderón podemos comprobar como los valores más elevados corresponden a los años más húmedos, sobre todo en dos de los tres

\section{Riqueza de grupos}

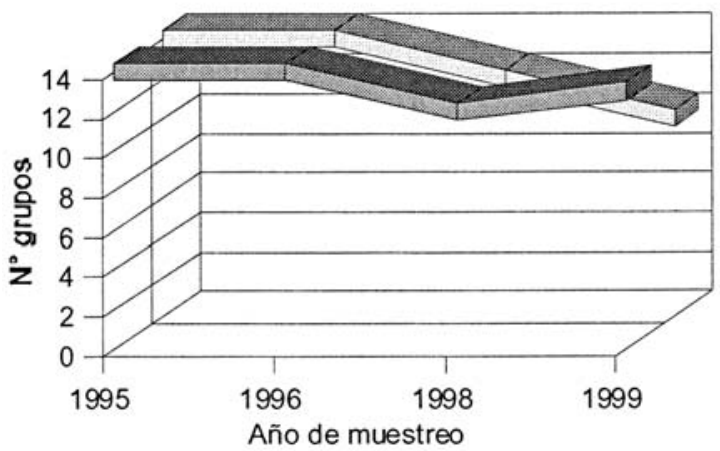

\section{Calderón $\square$ La Laguna}

Figura 2. Riqueza de grupos por año y laguna estudiada. Group richness per year and site. hábitats, mientras que La Laguna presenta valores similares durante los cuatro años.

Respecto a la riqueza de grupos en cada una de las dos lagunas y a lo largo de todo el periodo de estudio, debemos señalar que en Calderón, durante los dos primeros años, el número de taxones encontrados es mayor que el correspondiente a los años con menor nivel de precipitaciones, ocurriendo lo mismo en La Laguna.

En las figuras 3 y 4 se representan los dendrogramas correspondientes a las clasificaciones jerárquicas de los taxones encontrados en los muestreos "Biótopos-Año". En el primero
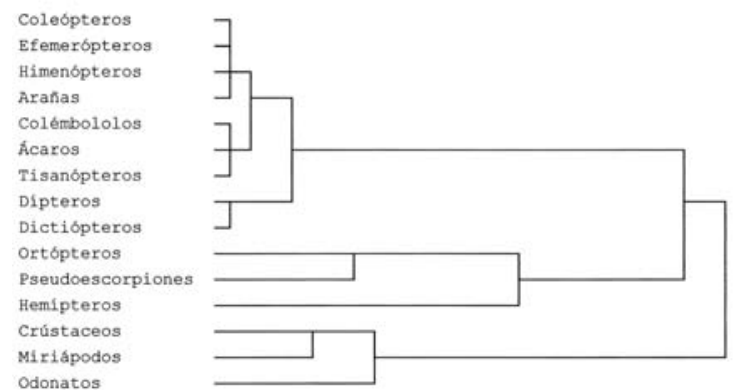

Figura 3. Dendrograma correspondiente a la clasificación jerarquica "artrópodos-año" en Calderón. Cluster dendrogram corresponding to the hierarchical classification "arthropods year " in Calderón. 

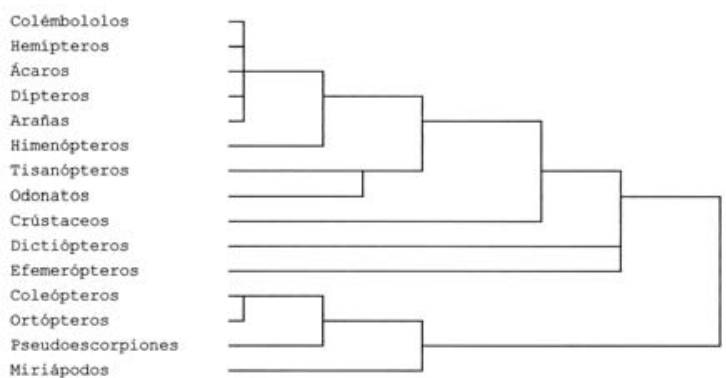

Figura 4. Dendrograma correspondiente a la clasificación jerarquica "artrópodos-año" en La Laguna. Cluster dendrogram corresponding to the hierarchical classification "arthropods-year " in La Laguna.

de ellos, correspondiente a los años más húmedos, se distinguen tres agrupamientos, el primero de los con valores más bajos de correlación que agrupa a los taxones de artrópodos edáficos más numerosos; el segundo con valores más elevados que solo relaciona a tres órdenes poco representativos y el tercero con una correlación intermedia entre los dos anteriores, constituido por los artrópodos edáficos que requieren un cierto grado de humedad para su desarrollo.

Al observar la correlación de los años secos en la figura 4, observamos como los agrupamientos se modifican totalmente, distinguiéndose dos agrupaciones, la primera con valores muy elevados de correlación, en la que encontramos la mayoría de los grupos estudiados y una segunda en la que solo aparecen cuatro órdenes, con características ecológicas diferentes.

En la figura 5 se muestra el dendrograma obtenido a partir del análisis de cluster realizado a la matriz de grupos obtenidos en las dos lagunas, durante los cuatro años. Se observan dos agrupamientos, que se identifican uno claramente con La Laguna y otro con Calderón, exceptuando los muestreos pertenecientes a 1999 de Calderón que se agrupan con los de La Laguna, esto puede ser debido a que las frecuencias, en los tres hábitats (olivar, viña y liega) y las densidades totales observadas en este hábitat durante el año indicado para los colémbolos y ácaros son muy similares a los de La Laguna.

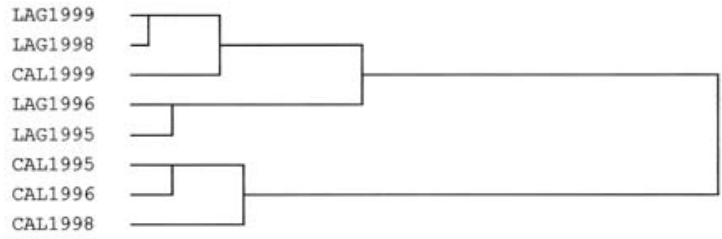

Figura 5. Dendrograma correspondiente a la clasificación jerarquica "laguna-año". Cluster dendrogram corresponding to the hierarchical classification "lagoon-year".

\section{DISCUSIÓN}

Tal y como señalamos en el apartado anterior, las zonas naturales presentan mayor número de artrópodos edáficos que los hábitats modificados, corroborando esto lo observado en otros estudios sobre entomofauna edáfica (Ruiz, et al., 1992; García Ruiz, 2001).

Los ácaros y colémbolos presentan un porcentaje de abundancia similar en las dos hábitats estudiados, esto puede atribuirse a mayores semejanzas en función de factores como la humedad, temperatura o tipo de suelo, y dentro de cada grupo, tal como señalamos anteriormente los colémbolos muestran una clara preferencia por las zonas naturales, mientras que los ácaros e himenópteros prefieren las zonas cultivas, pudiendo deberse esto a las condiciones ambientales favorables que se generan para el desarrollo de estos artrópodos, debido a los tratamientos sufridos por los suelos para estos cultivos.

Por el contrario, respecto a los grupos que hemos encontrado con menor número de individuos, cabe destacar los casos de las arañas y miriápodos, en los que aunque el número de ejemplares recogidos es pequeño, con respecto a otros grupos y los hemos encontrado durante la mayor parte del año, sobre todo en La Laguna.

Con los resultados obtenidos en los dendrogramas que reflejan las clasificaciones jerárquicas de los taxones estudiados en los diferentes muestreos, podemos señalar que posiblemente los artrópodos edáficos cuando existe un aumento de la humedad en el medio edáfico en que se encuentran, pueden desarrollarse por sí 
solos, mientras que en las épocas en las que la humedad es menor, pueden agruparse en microhábitats con mejores condiciones ambientales para su desarrollo, tal como señalamos en el estudio de la distribución horizontal.

\section{CONCLUSIONES}

Con este estudio podemos señalar que el incremento de humedad en el medio edáfico de las lagunas temporales produce efectos sobre la edafofuna de artrópodos de la zona, provocando el incremento de individuos de determinados grupos, favorecidos por las condiciones de humedad que se generan.

Las épocas con menor nivel de precipitaciones condicionan el desarrollo de la mayoría de los grupos de artrópodos edáficos, tal como hemos puesto de manifiesto con el análisis desarrollado.

\section{BIBLIOGRAFÍA}

ASCASO, J. 1984. Utilización de trampas de caída en dos comunidades forestales de la región medi- terránea: observaciones. Actas II Congreso Ibérico de Entomología, 1: 497-528.

ASCASO, J. 1986. Estudio cuantitativo de poblaciones a partir de muestreos indirectos: aplicación a comunidades de Artrópodos en dos bosques del Montseny. Tesis Doctoral, Universidad Autónoma de Barcelona. 479 pp.

CANCELA DA FONSECA, J. P. 1966. L'outil statistique en biologie du sol. III. Indices d'interes ecologique. Rev. Ecol. Biol. Sol, 3(3): 381-407.

HAGVAR, S. 1982. Collembolla in Norwegian coniferous forest soils. I. Relations to plant communities and soil fertility. Pedobiología, 24: 255296.

GARCÍA RUIZ, A. 2001. Estudio de los efectos de un incendio sobre las poblaciones de miriápodos. Ecología, 15: 269-273.

POURSIN, J. M. \& J. F. PONGE. 1984. Étude des peuplementes de microarthropodes (Insectes Collemboles et Acariens Oribates) dans trois humus forestiers acides de la Fôret d'Orléans (Loiret, France). Pedobiologia, 26: 403414.

RUIZ, M., E. PEREZ, P. GUERRA y J. C. SIMÓN. 1992. Estudio comparativo de la composición de artrópodos edáficos en zonas con vegetación natural y repobladas del Sistema Central (España). Bolm. Soc. Port. Ent., 2: 587-596. 